\title{
Impact of tobramycin on the performance of microbial fuel cell
}

\author{
Wenguo $\mathrm{Wu}^{1,2^{*}}$, Keaton Larson Lesnik${ }^{2}$, Shoutao $\mathrm{Xu}^{2}$, Luguang Wang ${ }^{2}$ and Hong Liu ${ }^{2}$
}

\begin{abstract}
Background: The release of antibiotics into aquatic environments has made the treatment of wastewater containing antibiotics a world-wide public health problem. The ability of microbial fuel cells (MFCs) to harvest electricity from organic waste and renewable biomass is attracting increased interest in wastewater treatment. In this paper we investigated the bioelectrochemical response of an electroactive mixed-culture biofilm in MFC to different tobramycin concentrations.

Results: The electroactive biofilms showed a high degree of robustness against tobramycin at the level of $\mu \mathrm{g} / \mathrm{L}$. The current generation responses of the biofilms were affected by the presence of tobramycin. The inhibition ratio of the MFC increased exponentially with the tobramycin concentrations in the range of 0.1-1.9 g/L. The bacterial communities of the biofilms vary with the concentrations of tobramycin, the equilibrium of which is critical for the stability of electroactive biofilms based-MFC.

Conclusions: Experimental results demonstrate that the electroactive biofilm-based MFC is robust against antibiotics at the level of $\mu \mathrm{g} / \mathrm{L}$, but sensitive to changes in antibiotic concentration at the level of $\mathrm{g} / \mathrm{L}$. These results could provide significant information about the effects of antibiotics on the performance MFC as a waste-treatment technology.
\end{abstract}

\section{Background}

Antibiotics, one of the important group of pharmaceuticals in human and veterinary medicine, are widely used in the prevention and treatment of diseases and have been detected in various aquatic environments, for example, wastewater, surface water, ground water and drinking water [1-3]. Therefore, the release of antibiotics to the aquatic environment as well as its related environmental issues and public health problems have attracted great attention. Biological treatment, the use of bacteria and other microorganisms to remove contaminants by assimilating or oxidizing them, is still regarded as the most common and economical approach for the treatment of contaminants in wastewater [4]. Traditionally aerobic treatment consumes large amounts of electrical energy for aeration [5]. Anaerobic treatment is generally only suitable for high-strength wastewater streams typically produced by industry [6].

\footnotetext{
* Correspondence: wuwenguo@hqu.edu.cn

${ }^{1}$ College of Chemical Engineering, Huaqiao University, Xiamen 361021, China

${ }^{2}$ Department of Biological and Ecological Engineering, Oregon State University, Corvallis, OR, USA
}

Microbial fuel cell (MFC) as a device capable of harvesting electricity from organic waste and renewable biomass, has attracted great interest for wastewater treatment [5]. There are various reports about MFCs for biodegradable organics as substrates, for example, glucose, lactate, sucrose, domestic wastewater, brewery wastewater, whey wastewater and starch processing wastewater [7-11]. Recently MFC technology used for removing toxic and recalcitrant contaminants as substitute substrates in wastewater with much higher chemical oxygen demand (COD) removal efficiencies has drawn great attention $[4,12]$. The treatment capacity of MFC technology was mainly dependent on the performance of the MFC. In contrast to planktonic cells, wastewater-derived electroactive biofilms show less susceptibility to toxins making MFCs promising for application in the wastewater treatment field [13]. Furthermore, the goal of wastewater treatment is COD removal. However, the question that is still largely unaddressed is the effect of the toxins on the performance of electroactive biofilms-based MFC accomplishing removal of COD in pharmaceutical wastewater treatment.
C Biomed Central

(c) 2014 Wu et al.; licensee BioMed Central Ltd. This is an Open Access article distributed under the terms of the Creative Commons Attribution License (http://creativecommons.org/licenses/by/2.0), which permits unrestricted use, distribution, and reproduction in any medium, provided the original work is properly credited. The Creative Commons Public Domain Dedication waiver (http://creativecommons.org/publicdomain/zero/1.0/) applies to the data made available in this article, unless otherwise stated. 
Tobramycin, an aminoglycoside produced by the bacterium Streptomyces tenebrarius, is commonly used because of its enhanced effectiveness against infections with the opportunistic pathogen Pseudomonas aeruginosa [14]. Tobramycin targets the decoding aminoacyl site on the $16 \mathrm{~S}$ ribosomal RNA, induces miscoding during translation and cell death ensues $[15,16]$. Herein, the impact of tobramycin on the performance of electroactive biofilms-based MFC was studied. The mixed-culture microorganism community was harvested from wastewater and formed electrochemically active biofilms on the anode generating steady current. An adaptation or change of the microbial community will influence the biofilm structure and the stability of electroactive biofilms-based MFC [17]. Hence, the microbial community of the anodic biofilm was analyzed using pyrosequencing and changes of the microbial diversity with different concentrations of tobramycin were determined by denaturing gradient gel electrophoresis (DGGE).

\section{Results and discussion}

\section{Effects of tobramycin on the performance of MFCs}

Electrochemically active biofilms were enriched on the anode of MFC, whereupon a repeatable and steady output current in the range of 1.2-1.4 $\mathrm{mA}$ was developed at the resistance of $300 \Omega$ without antibiotic (batch 1 in
Figure 1). Tobramycin was added into the MFC when the current was stabilized in batch 2 . There was also no obvious immediate current decrease after addition of tobramycin at different concentrations (batch 2). However, subsequent batches exhibited distinct current profiles which were more notable at increased tobramycin concentrations. At concentrations of $0.2,0.5$, and $1 \mathrm{mM}$, inhibition was only exhibited at the beginning of the batch, after which a stable maximum current was maintained. Obvious inhibitions were observed in the MFC with tobramycin concentration of $2 \mathrm{mM}$ at batch 3 and the MFC showed significant inhibition as the concentration increased to $4 \mathrm{mM}$. However, for the MFCs with tobramycin concentrations of 2 and $4 \mathrm{mM}$, current recovered 4 and 6 batches post antibiotic addition. These results suggested that some microbial species in the biofilm directly or indirectly facilitating current generation in the biofilm could be recovered from those community members who were not killed by the given antibiotic dose and exposure time, reflected by the recovering stable current for a few batches. The current recovery of MFCs indicated the resistance of the microbial biofilms against the presence of tobramycin in the range of $0.2-4 \mathrm{mM}$, corresponding to a concentration range of about 0.1-1.9 g/L. Furthermore, pharmaceuticals have been found in surface waters and wastewaters at levels of up to a few $\mu \mathrm{g} / \mathrm{L}$ [18]. It suggested

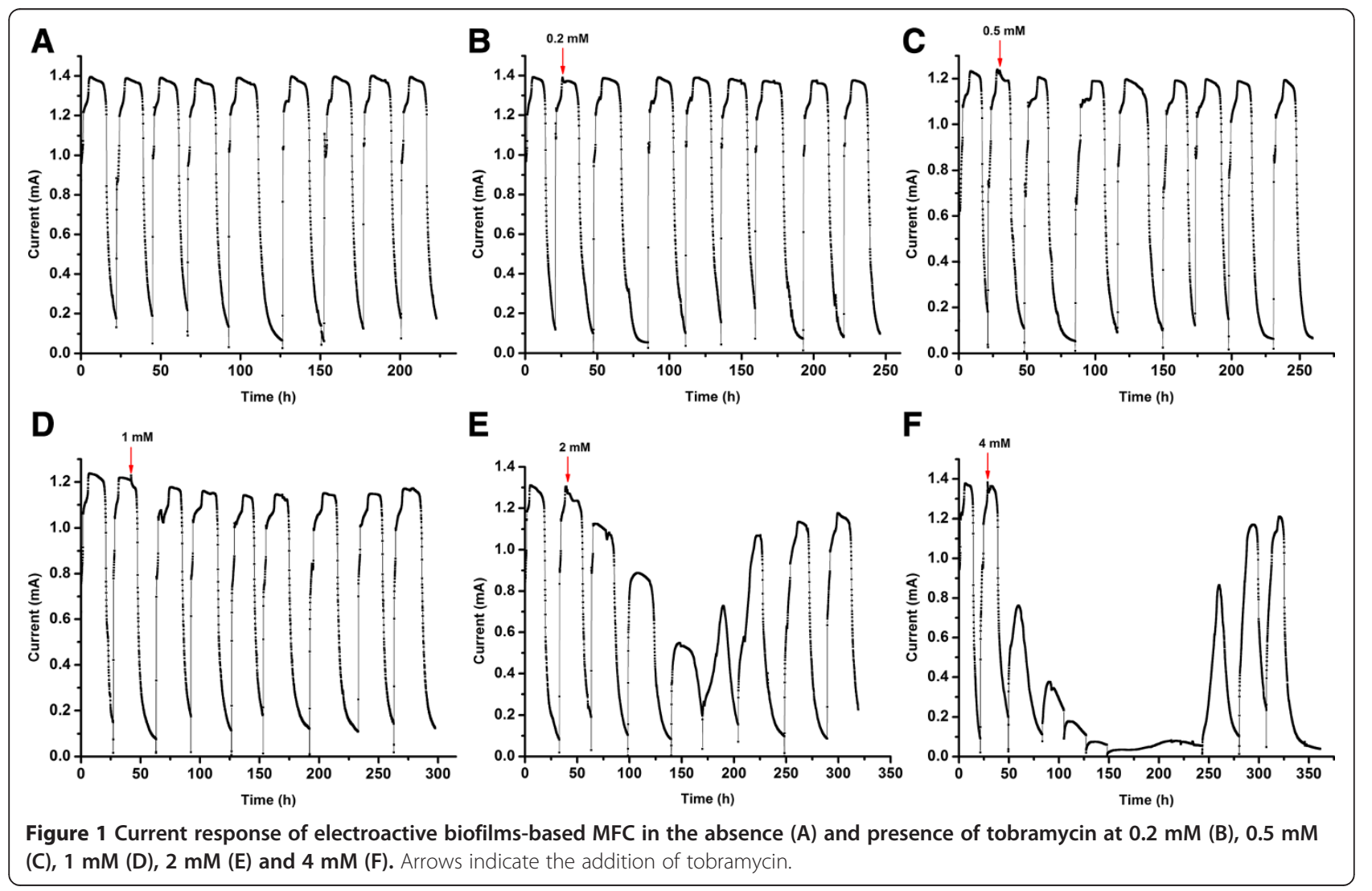


that there is no response of the biofilm at all in wastewater treatment containing antibiotics in this range. A similar result was also reported that no effect was observed in biofilms-based MFCs in the presence of toxins which were at concentrations an order of magnitude higher than average concentrations in wastewaters [13].

\section{Inhibition ratio of MFCs correlated to tobramycin concentrations}

In order to further explore the electroactive biofilms against antibiotics at levels of $\mathrm{g} / \mathrm{L}$, we compared the inhibition ratios of electroactive biofilms-based MFCs in the presence of different concentrations of tobramycin. As shown in Figure 2, tobramycin showed an exponential relationship of the inhibition ratio with the antibiotic concentrations. The regression equation is:

$$
\mathrm{y}=-10.3\left(1-e^{0.4 \mathrm{x}}\right), \mathrm{R}^{2}=0.99998
$$

This indicated that the reaction taking place on the anode of the MFC would be a combination of biofilm kinetics and electrochemical kinetics. The changing of kinetic inhibition of microorganisms in the biofilm would be one reason of the non-linear correlation between tobramycin and inhibition ratio [19]. The inhibition ratio of tobramycin drastically increased from $0.2 \%$ to $42.6 \%$ as the antibiotic concentrations were increased from $0.2 \mathrm{mM}$ to $4 \mathrm{mM}$. The tobramycin concentration of $4 \mathrm{mM}(1870 \mathrm{mg} / \mathrm{L})$ is three orders of magnitude higher than the reported minimal biofilm eliminating concentration (MBEC) of E. coli $(2 \mathrm{mg} / \mathrm{L})$ measured by the traditional colony-forming unit (CFU) counting method [20]. The inhibition of the biofilm at the tobramycin

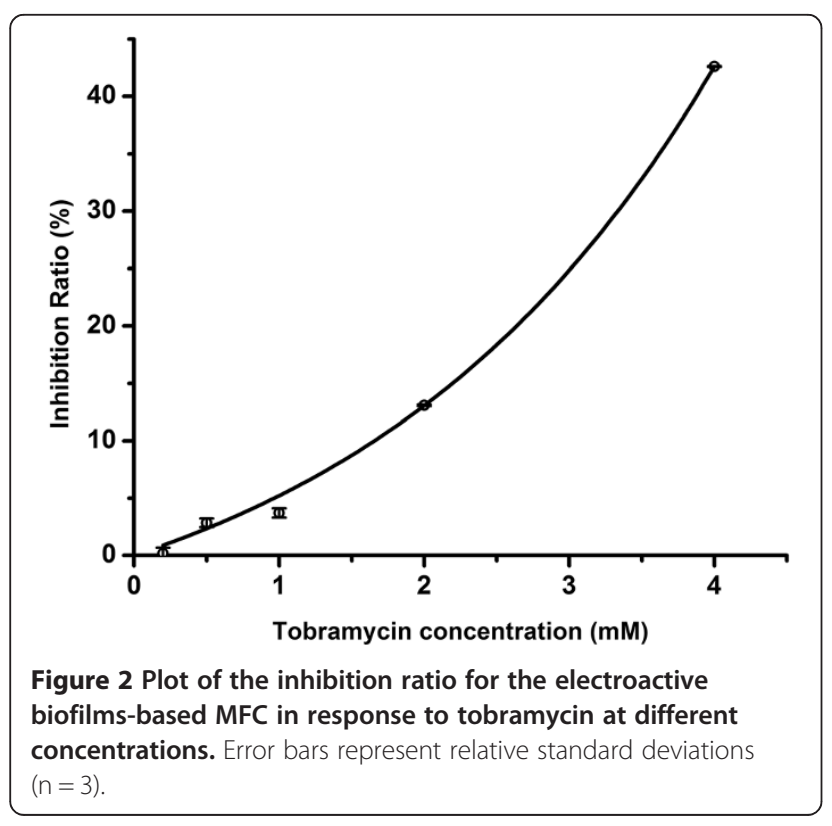

concentration of $4 \mathrm{mM}$ may lead to significantly reduced substrate oxidation rates and subsequent decreased voltage outputs and substrate consumption rates. Experimental results indicated that the electroactive biofilmbased MFC was robust against antibiotics at the level of $\mu \mathrm{g} / \mathrm{L}$ but sensitive to changes in antibiotic concentration at the level of $\mathrm{g} / \mathrm{L}$. Kim et al. also reported similar results and suggested a novel biomonitoring system using MFCs for the detection of several toxins at the level of $\mathrm{mg} / \mathrm{L}$ [21].

\section{MFCs exposure to continuously increasing concentration of tobramycin}

To test the continuous effect of tobramycin on MFCs, the performance of the electroactive biofilms-based MFCs in the presence of increasing concentrations of tobramycin was observed (Figure 3). Similar with the result of the MFCs in Figure 1, the current decreased in the batch immediately after the injection of tobramycin and showed greater reduction with the increase of concentration. However, after the addition of tobramycin at the concentration of $6 \mathrm{mM}$, the significantly inhibited current still recovered to a stable current. It was suggested that some biofilm community members died and some survived during each batch with tobramycin, and then the community regrew in the subsequent batches with no tobarmycin. Moreover, the balance between competition and commensalism in the microbial community of the biofilms in the presence of tobramycin could be one of the reasons for the recovery of a stable current. These results clearly showed the effects of an intermittent shock load and the ability of the anode community to recover from a shock load in the presence of tobramycin. It provides significant information about antibiotics effects on MFC as a wastetreatment technology.

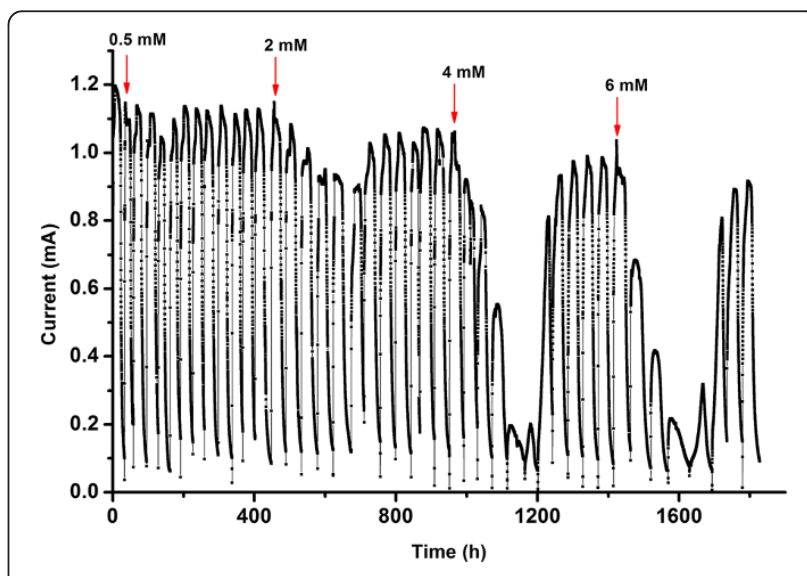

Figure 3 Current response of electroactive biofilms-based MFC in the presence of continuously increasing concentrations of tobramycin. Arrows indicate the addition of tobramycin. 


\section{Effect of tobramycin on the microbial community}

The effects of tobramycin concentration on mature acetate-fed MFC anode biofilm communities were analysed using a combination of pyrosequencing and DGGE. Pyrosequencing of the control community (before the addition of tobramycin) yielded 1317 high-quality $16 \mathrm{~s}$ rRNA gene reads and following taxonomic assignment revealed the community to be primarily composed of 3 phyla, Proteobacteria (52.2\%), Synergistetes (27.8\%), and Firmicutes (13.7\%) (Figure 4). Geobacter spp. made up $38.9 \%$ of the community while Aminiphilus spp represented $27.8 \%$ of 16 s rRNA reads. These populations correspond to band 4 and band 5, respectively (Figure 5). This community structure is consistent with previous analyses of a community maintained in the same laboratory [22]. Geobacter spp. are well-known for their capability of colonizing and actively respiring anodes resulting in significant current generation [23-25], while Aminiphilus spp. likely play a role as the primary fermentative bacteria, recycling peptones and amino acids into acetate and hydrogen [26]. Fermenation-produced acetate could then be ultilized by anode-respiring species such as Geobacter spp. The third most common genera, Acetoanaerobium, fits the role of a homo-acetogenic hydrogen scavenger [27], and along with Geobacter and Aminiphilus is expected to be involved in a proposed syntrophic interactions that leads to high power densities in MFCs [28]. Members of the Bacteroidia class were also prominent in on the DGGE gel (Figure 5) (bands $1 / 2$ ), yet only represented $2.1 \%$ of the $16 \mathrm{~s}$ pyrosequencing reads with no clear picture of the role they in the present community.

Results following antibiotic introduction show that the microbial communities varied with the increase of tobramycin concentration (Figure 5). As shown in Figure 5 , band 4 , band 5 , and band 6 disappeared at a tobramycin concentration of $4 \mathrm{mM}$, as tobramycin, being most effective against gram-negative bacteria, was able to reduce populations of Geobacter spp., Aminiphilus spp., and Acetoanaerobium spp. The presence of these members within the community was associated with high power outputs and following their loss at increased antibiotic concentrations concurrent decreases in power outputs were observed (Figure 1). The communities disturbed with high concentrations of tobramycin were the most diverse with unknown bands emerging (Figure 5) (band 3 and 7). Though some similarities can be seen between communities exposed to $3 \mathrm{mM}$ and $4 \mathrm{mM}$ tobramycin, such as the emergence of band 3, other bands were only prominent in one of the treatments (band 7). This suggests that though tobramycin-resistant bacteria were enriched at higher concentrations, it is likely varying community structures will emerge following antibiotic-disruption in contrast to the stability of the community under acetate-fed conditions.

\section{Conclusions}

An electroactive biofilm based-MFC was developed and the electrochemical response of different concentrations of tobramycin on the electroactive mixed-culture biofilms was studied along with the effect to community structure. The electroactive biofilms showed a high degree of robustness against tobramycin at the level of $\mu \mathrm{g} / \mathrm{L}$. The current generated by the electrochemically active biofilm decreased as the tobramycin concentration arrived in the range of 0.1-1.9 $\mathrm{g} / \mathrm{L}$ and the inhibition ratio increased with the increase of tobramycin concentration. The bacterial communities of the biofilms varied with the concentrations of

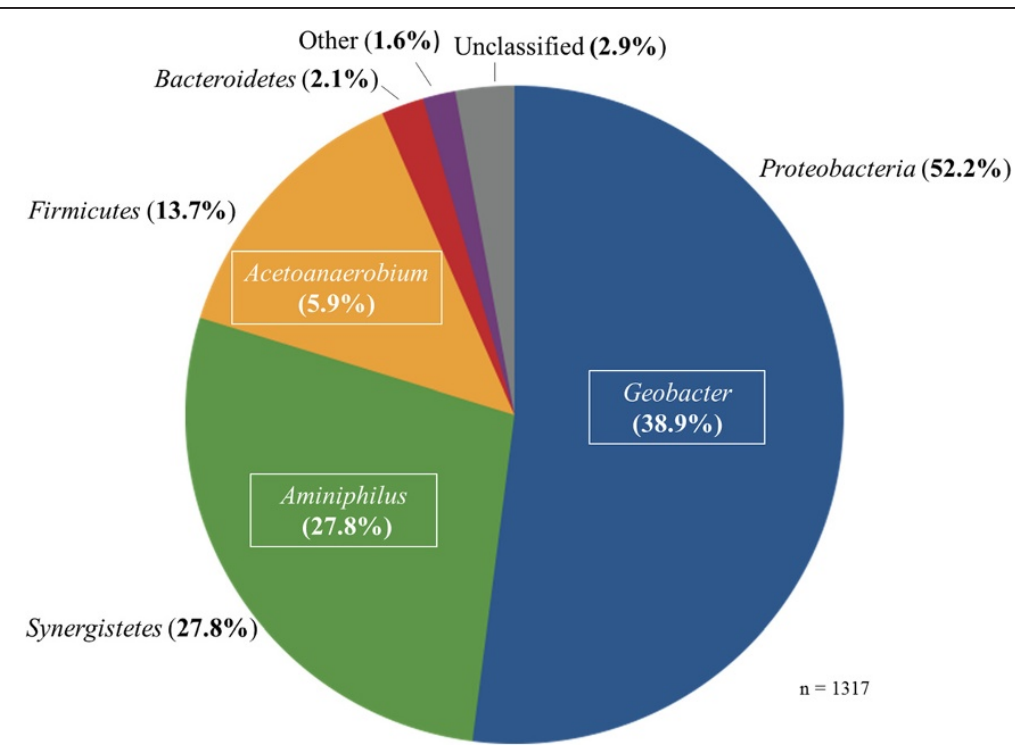

Figure 4 Core microbiome of electroactive biofilms-based MFC with no antibiotic addition (percent of total 16S rRNA reads). 


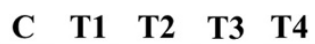
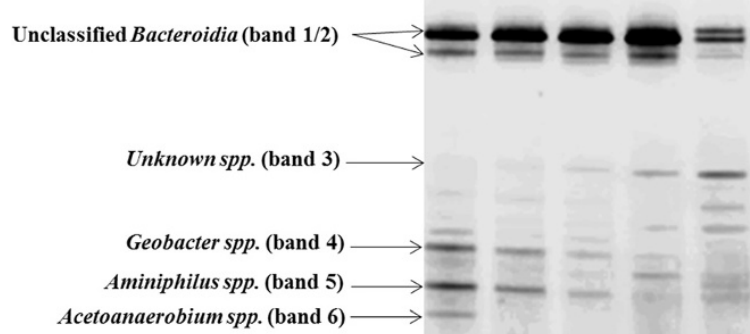

Unknown spp. (band 7) $\rightarrow$

Figure 5 PCR-DGGE analysis of 16S rDNA extracted from the electroactive biofilms-based MFC without tobramycin (lane C) and in the presence of tobramycin at concentrations of $0.5 \mathrm{mM}$ (lane T1), 1 mM (lane T2), 2 mM (lane T3) and 4 mM (lane T4).

tobramycin, the equilibrium of which is critical to the stability of electroactive biofilm based-MFCs. These results could provide significant information about the effects of antibiotics on the performance MFC as a waste-treatment technology. In the future, studies on various other microorganisms and antibiotics like sulfadiazine, enoxacin and bacitracin will provide more conclusive results for the treatment of wastewater from pharmaceutical industries.

\section{Methods}

\section{Construction of electroactive biofilms-based MFCs}

A single-chamber MFC was constructed as described previously [29]. Briefly, the anode and cathode were placed in parallel on the opposite sides of the chamber $(13 \mathrm{~mL})$ with a distance of $1.7 \mathrm{~cm}$. Non-wet proofed carbon cloth (type A, E-TEK, Somerset, NJ, USA; $2 \mathrm{~cm}^{2}$ ) were used as the anode without any treatment. Wet-proofed (30\%) carbon cloth (type B, ETEK, Somerset, NJ, USA; $7 \mathrm{~cm}^{2}$ ) was coated with carbon/poly (tetrafluoroethylene) (PTFE) layers on the air-facing side and platinum $\left(0.5 \mathrm{mg} / \mathrm{cm}^{2}\right.$ cathode area) with Nafion as binder on the water-facing side, and used as the cathode.

\section{Operation of electroactive biofilms-based MFCs}

Medium used for enrichment and operation of the microbial fuel cell was prepared as previously described [30]. Sodium acetate $(60 \mathrm{mM})$ was used as the carbon resource. The medium $(8 \mathrm{ml})$ in the MFC was inoculated with $5 \mathrm{~mL}$ of electrochemically active mixed-culture microorganisms, a mixture of gram-positive and gram-negative microorganisms reported in our previous study [30]. The electroactive biofilms-based MFC was monitored by a data acquisition system (2700, Keithly, Cleveland, OH, USA), the acetate medium solution was refreshed after each batch until a stable power output was obtained at an external resistance of $300 \Omega$. When the standard deviation of maximal voltage in the each batch after three batches was within $\pm 5 \%$, 2.6-78 $\mu \mathrm{l}$ of the tobramycin stock solution $(1000 \mathrm{mM})$ was added to the MFC medium to obtain final concentrations in the range of $0.2-6 \mathrm{mM}$ for a single batch, subsequent voltages were then recorded. The following post-antibiotic batches were replaced with the fresh acetate medium solution without tobramycin. Each concentration was run in triplicate.

\section{Microbial community analysis}

Biofilms were separated from the anodes of MFCs treated with different antibiotic concentrations for 20 days (around 10 batches). Bacterial genomic DNA was extracted from the biofilm samples using the DNeasy tissue Kits (Qiagen, CA, USA) according to the manufacturer's instructions.

The control community with no antibiotic addition was then prepared for pyrosequencing. Primers developed to target the hyper-variable V4 region of the $16 \mathrm{~S}$ rRNA gene (Cole et al. [31]). The 454 adapter sequence $\left(5^{\prime}-3^{\prime}\right)$ CCTATCCCCTGTGTGCCTTGGCAGTC the forward primer AYTGGGYDTAAA GNG (Escherichia coli position 563-577). The reverse primers were composed of the adapter sequence followed by the reverse primer sequence, CCGTCAATTCMTTTRAGT (E. coli 907-924). Twenty-five microliter PCR reaction volumes were used for optimization followed by $50 \mu \mathrm{l}$ amplification reactions. A high-fidelity Taq polymerase (Invitrogen Platinum) was used with along with $\mathrm{MgSO}_{4}(2.5 \mathrm{mM})$, vendor supplied buffer, BSA $(0.1 \mathrm{mg} / \mathrm{ml})$, dNTPs $(250 \mu \mathrm{M})$, and primers $(1 \mu \mathrm{M})$. An initial 3-min step at $95^{\circ} \mathrm{C}$ was followed by 27 cycles of $95^{\circ} \mathrm{C}(45 \mathrm{~s}), 57^{\circ} \mathrm{C}(45 \mathrm{~s})$, and $72^{\circ} \mathrm{C}(1 \mathrm{~min})$ with a final $3 \mathrm{~min}$ extension at $72^{\circ} \mathrm{C}$. PCR products were agarose gel purified ( $2 \%$ metaphor in TAE) and bands were extracted with a QIAquick Gel Extraction Kit (Qiagen, Valencia, CA). Gel extracted material was further purified with a Qiagen PCR Cleanup kit and AMPure XP magnetic beads. Quantification of purified PCR product was performed using a Qubit fluorom- eter (Invitrogen, Carlsbad, CA) and qPCR (ABI PRISM 7500 FAST Detection System). Following quantification, libraries were pooled into equimolar amounts. Emulsion PCR and sequencing was performed on a 454 GS Junior pyrosequencer (Roche, Nutley, NJ, USA) at the Center for Genome Research and Biocomputing (CGRB), Oregon State University using titanium reagents and procedures consistent with protocols for unidirectional amplicon sequencing.

Initial quality filtering was performed using MOTHUR, alignment was performed using MUSCLE and subsequent taxonomic identification done using RDP Classifier at an 
$80 \%$ confidence level [31-33]. Classifier results were then used for community analysis.

All samples were also subject to analysis through DGGE. After nesting 16S ribosomal DNA (rDNA) by using a pair of universal primers: $27 \mathrm{~F}$ (5' -AGAGTTTG ATCMTGGCTCAG-3') and 1492R (5' -GGTTACCTT TGTTACGACTT-3') [34]. The universal primer set $357 \mathrm{~F}$ GC (5'-GC-clamp-CCTACGGGAGGCAGCAG-3') and 518R (5' - ATTACCGCGGCTGCTGG-3') (Invitrogen, Carlsbad, CA, USA) was used to amplify the V3 region of bacteria $16 \mathrm{~s}$ rDNA from the extracted genomic DNA. PCR amplification and cycling were performed in a thermocycler (Thermo hybaid, MBS 0.2G, Thermo, MA, USA). DGGE of the PCR products was carried out in a DcodeTM Universal Mutation Detection System (Bio-rad Laboratories, Hercules, CA, USA). Prominent DGGE bands were then excised from the gel and their products amplified using the same PCR system. Amplified products from these bands were then submitted to CGRB for sanger sequencing. Pyrosequencing and DGGE results could then be correlated, allowing for a more comprehensive community analysis.

\section{Data deposition}

Genomic datasets were deposited in the NCBI sequence read archive under accession number. The genomic project can also be accessed in NCBI under Genome Project ID PRJNA252648. (accession, http://www.ncbi.nlm.nih. gov/bioproject?term=PRJNA252648).

\section{Calculation}

The inhibition ratio (I) was calculated as I $(\%)=100 \times$ $\left(A_{M 1}-A_{M 2}\right) / A_{M 1}$, where $A_{M 1}$ was the maximal current in the batch before tobramycin addition, $\mathrm{A}_{\mathrm{M} 2}$ was the maximal current in the following batch after addition of tobramycin.

\section{Competing interests}

The authors declare that they have no competing interests.

\section{Authors' contributions}

WW carried out the operation of electroactive biofilms-based MFC, participated in the microbial community analysis and drafted the manuscript. $\mathrm{KLL}$ carried out the microbial community analysis. SX participated in the construction of MFC and helped to revise the manuscript. LW participated in microbial community analysis. HL participated in its design and coordination and helped to draft the manuscript. All authors read and approved the final manuscript.

\section{Acknowledgements}

This work was supported by the U.S. National Science Foundation (CBET 0955124), National Natural Science Foundation of China (NSFC 81301290), Quanzhou Science and Technology Plan Project (2013Z24) and Research Foundation for High-level Talents of Huaqiao University (12BS207). We thank Mark Dasenko at the Oregon State University Center for Genome Research and Biocomputing (CGRB) for sequencing support. Wenguo Wu would like to thank the China Scholarship Council for supporting her study at Oregon State University.
Received: 26 November 2013 Accepted: 15 June 2014 Published: 4 July 2014

\section{References}

1. Perret D, Gentili A, Marchese S, Greco A, Curini R: Sulphonamide residues in Italian surface and drinking waters: a small scale reconnaissance. Chromatographia 2006, 63:225-232.

2. Stolker AAM, Niesing W, Hogendoorn EA, Versteegh JFM, Fuchs R, Brinkman UAT: Liquid chromatography with triple-quadrupole or quadrupole-time of flight mass spectrometry for screening and confirmation of residues of pharmaceuticals in water. Anal Bioanal Chem 2004, 378:955-963.

3. Ye ZQ, Weinberg HS, Meyer MT: Trace analysis of trimethoprim and sulfonamide, macrolide, quinolone, and tetracycline antibiotics in chlorinated drinking water using liquid chromatography electrospray tandem mass spectrometry. Anal Chem 2007, 79:1135-1144.

4. Wen Q, Kong FY, Zheng HT, Yin JL, Cao DX, Ren YM, Wang GL: Simultaneous processes of electricity generation and ceftriaxone sodium degradation in an air-cathode single chamber microbial fuel cell. J Power Sources 2011, 196:2567-2572.

5. Rozendal RA, Hamelers HVM, Rabaey K, Keller J, Buisman CJN: Towards practical implementation of bioelectrochemical wastewater treatment. Trends Biotechnol 2008, 26:450-459.

6. Liu H, Ramnarayanan R, Logan BE: Production of electricity during wastewater treatment using a single chamber microbial fuel cell. Environ Sci Technol 2004, 38:2281-2285.

7. Feng $Y$, Wang $X$, Logan $B E$, Lee $H$ : Brewery wastewater treatment using air-cathode microbial fuel cells. Appl Microbiol Biotechnol 2008, 78:873-880.

8. Lu N, Zhou SG, Zhuang L, Zhang JT, Ni JR: Electricity generation from starch processing wastewater using microbial fuel cell technology. Biochem Eng J 2009, 43:246-251.

9. Min B, Logan BE: Continuous electricity generation from domestic wastewater and organic substrates in a flat plate microbial fuel cell. Environ Sci Technol 2004, 38:5809-5814.

10. Nasirahmadi S, Safekordi AA: Enhanced electricity generation from whey wastewater using combinational cathodic electron acceptor in a two-chamber microbial fuel cell. Int J Environ Sci Technol 2012, 9:473-478.

11. Wu WG, Bai LL, Liu X, Tang ZM, Gu ZZ: Nanograss array boron-doped diamond electrode for enhanced electron transfer from Shewanella loihica PV-4. Electrochem Commun 2011, 13:872-874.

12. Wen $\mathrm{Q}$, Kong FY, Zheng HT, Cao DX, Ren YM, Yin JL: Electricity generation from synthetic penicillin wastewater in an air-cathode single chamber microbial fuel cell. Chem Eng J 2011, 168:572-576.

13. Patil S, Harnisch F, Schröder U: Toxicity response of electroactive microbial biofilms-a decisive feature for potential biosensor and power source applications. ChemPhysChem 2010, 11:2834-2837.

14. Goodman LS, Gilman A: Goodman and Gilman's The Pharmacological Basis of Therapeutics. New York: McGraw-Hill Professional Publishing; 1985.

15. Davies J, Davis BD: Misreading of ribonucleic acid code words induced by aminoglycoside antibiotics-effect of drug concentration. J Biol Chem 1968, 243:3312-3316.

16. Davis BD: Mechanism of bactericidal action of aminoglycosides. Microbiol Rev 1987, 51:341-350.

17. Sutherland IW: The biofilm matrix-an immobilized but dynamic microbial environment. Trends Microbiol 2001, 9:222-227.

18. Seifrtová M, Novaková L, Lino C, Pena A, Solich P: An overview of analytical methodologies for the determination of antibiotics in environmental waters. Anal Chim Acta 2009, 649:158-179.

19. Stein NE, Keesman KJ, Hamelers HVM, van Straten G: Kinetic models for detection of toxicity in a microbial fuel cell based biosensor. Biosens Bioelectron 2011, 26:3115-3120.

20. Ceri H, Olson ME, Stremick C, Read RR, Morck D, Buret A: The calgary biofilm device: new technology for rapid determination of antibiotic susceptibilities of bacterial biofilms. J Clin Microbiol 1999, 37:1771-1776.

21. Kim M, Sik Hyun M, Gadd GM, Joo Kim H: A novel biomonitoring system using microbial fuel cells. J Environ Monit 2007 9:1323-1328.

22. Lesnik LK, Liu H: Establishing a core microbiome in acetate-fed microbial fuel cells. Appl Microbiol Biot 2014, 98:4187-4196.

23. Bond DR, Lovley DR: Electricity production by Geobacter sulfurreducens attached to electrodes. Appl Environ Microbiol 2003, 69:1548-1555. 
24. Holmes DE, Bond DR, O'Neill RA, Reimers CE, Tender LR, Lovley DR: Microbial communities associated with electrodes harvesting electricity from a variety of aquatic sediments. Microb Ecol 2004, 48:178-190.

25. Kan JJ, Hsu L, Cheung ACM, Pirbazari M, Nealson KH: Current production by bacterial communities in microbial fuel cells enriched from wastewater sludge with different electron donors. Environ Sci Technol 2011, 45:1139-1146.

26. Díaz C, Baena S, Fardeau ML, Patel BKC: Aminiphilus circumscriptus gen. nov., sp. nov., an anaerobic amino-acid-degrading bacterium from an upflow anaerobic sludge reactor. Int I Syst Evol Microbiol 2007, 57:1914-1918

27. Sleat $R$, Mah RA, Robinson R: Acetoanaerobium noterae gen. nov., sp. nov.: an anaerobic bacterium that forms acetate from $\mathrm{H}_{2}$ and $\mathrm{CO}_{2}$. Int J Syst Evol Microbiol 1985, 35:10-15.

28. Parameswaran $\mathrm{P}$, Zhang $\mathrm{H}$, Torres $\mathrm{Cl}$, Rittmann BE, Krajmalnik-Brown R. Microbial community structure in a biofilm anode fed with a fermentable substrate: the significance of hydrogen scavengers. Biotechnol Bioeng 2010, 105:69-78.

29. Liu H, Cheng SA, Logan BE: Production of electricity from acetate or butyrate using a single-chamber microbial fuel cell. Environ Sci Technol 2005, 39:658-662.

30. Catal T, Li K, Bermek H, Liu H: Electricity production from twelve monosaccharides using microbial fuel cells. J Power Sources 2008, 175:196-200

31. Cole JR, Wang Q, Cardenas E, Fish J, Chai B, Farris RJ, Kulam-Syed-Mohideen AS, McGarrell DM, Marsh T, Garrity GM, Tiedje JM: The ribosomal database project: improved alignments and new tools for rRNA analysis. Nucl Acids Res 2009, 37:141-145.

32. Edgar RC: MUSCLE: multiple sequence alignment with high accuracy and high throughput. Nucl Acids Res 2004, 32:1792-1797.

33. Schloss PD, Westcott SL, Ryabin T, Hall JR, Hartmann M, Hollister EB, Lesniewski RA, Oakley BB, Parks DH, Robinson CJ, Sahl JW, Stres B, Thallinger GG, Van Horn DJ, Weber CF: Introducing mothur: open-source, platform-independent, community-supported software for describing and comparing microbial communities. Appl Environ Microbiol 2009, 75:7537-7541

34. Park HS, Kim BH, Kim HS, Kim HJ, Kim GT, Kim M, Chang IS, Park YK, Chang HI: A novel electrochemically active and Fe(III)-reducing bacterium phylogenetically related to Clostridium butyricum isolated from a microbial fuel cell. Anaerobe 2001, 7:297-306.

doi:10.1186/s12934-014-0091-6

Cite this article as: Wu et al:: Impact of tobramycin on the performance

of microbial fuel cell. Microbial Cell Factories 2014 13:91.

\section{Submit your next manuscript to BioMed Central and take full advantage of:}

- Convenient online submission

- Thorough peer review

- No space constraints or color figure charges

- Immediate publication on acceptance

- Inclusion in PubMed, CAS, Scopus and Google Scholar

- Research which is freely available for redistribution 